\title{
Dielectric Investigation of Glycine Phosphite Crystal
}

\author{
R. Czukwinski, Z. Czapla and R. Styrkowiec \\ Institute of Experimental Physics, University of Wrocław \\ pl. M. Borna 9, 50-204 Wrocław, Poland
}

(Received July 11, 2001)

\begin{abstract}
The complementary measurements of the dielectric dispersion in ferroelectric phase of glycine phosphite crystal at the frequency range of $200 \mathrm{~Hz}-$ $1 \mathrm{MHz}$ have been carried out. The analysis of the dielectric spectra of the low frequency relaxation process showed that it can be fitted by two Debye-type relaxators. The activation energy of the both processes has been calculated. The observed behaviour of the relaxators suggests that they are connected with a domain structure; one with the growth of the nuclei whereas the second with the domain wall movement.
\end{abstract}

PACS numbers: 77.80.Bh

\section{Introduction}

Glycine phosphite crystal $\left(\mathrm{NH}_{3}^{+} \mathrm{CH}_{2} \mathrm{COOH}\right)\left(\mathrm{H}_{2} \mathrm{PO}_{3}^{-}\right)=\mathrm{Gly} \cdot \mathrm{H}_{3} \mathrm{PO}_{3}$, abbreviated as GPI has been known for a long time [1]. At room temperature the structure of GPI is monoclinic with the space group $P 2_{1} / a$ and unit cell dimensions: $a=9.792 \AA, b=8.487 \AA, c=7.411 \AA, \beta=100.43^{\circ}, Z=4$ [1]. There exist two types of centrosymmetric, disordered hydrogen bonds with a double potential well for protons corresponding to $\mathrm{O}-\mathrm{O}$ distances of 2.482 and $2.518 \AA$.

Glycine phosphite crystals undergo a ferroelectric, continuous, second-order phase transition of the order-disorder type at $T_{\mathrm{c}}=224.7 \mathrm{~K}$ which was observed in dielectric [2, 3], differential scanning calorimetric (DSC) [4], and linear birefringence (LB) [5] measurements. The spontaneous polarization $P_{\mathrm{s}}$ is parallel to twofold $b$-axis and perpendicular to the infinite hydrogen bonded chains of the phosphite anions directed along the crystallographic $c$-axis. The value of the spontaneous polarization obtained from the hysteresis loop measurement was equal to about $5 \times 10^{-2} \mathrm{C} \mathrm{m}^{-2}$ at $170 \mathrm{~K}$. The symmetry of ferroelectric phase is expected to be $P 2_{1}$ [2]. The very large isotopic effect $(\approx 98 \mathrm{~K})$ found in GPI [4] and a significant effect of external pressure on temperature of the phase transition $T_{c}$ give 
evidences that the ordering of protons play a very important role in the molecular mechanism of phase transition [6,7]. The ultrasonic studies of the phase transition in GPI crystals indicate a pseudoproper character of the ferroelectric phase transition [8].

The infrared and Raman spectra [4] indicate more complex nature of the phase transition. There is a suggestion that ordering of the protons is coupled to the motion of the glycine cations. Recently, the results of the dynamics of the glycine molecules in GPI crystals using the proton NMR technique have been reported [9]. It is concluded that the rotation about the long axis of the glycine molecule is a type of motion, which influences the phase transition in GPI crystal.

The dielectric dispersion studies give complex information about the microscopic nature of the phase transition. Up to now there are several works in which the results of the dielectric dispersion studies for GPI and deuterated glycine phosphite (DGPI) crystals are reported $[4,10,11]$. Dielectric dispersion investigated in the frequency range of $100 \mathrm{~Hz}-27 \mathrm{GHz}$ showed a single relaxation time (Debye-type relaxation) in paraelectric phase and the two well-distributed relaxational processes in the ferroelectric phase; the first one in the high frequency range - ferroelectric dipolar dispersion (of the same nature as in the paraelectric phase) and the second one, observed only below $T_{\mathrm{c}}$, the low frequency relaxational process probably related to the domain structure appearance and domain wall motion. This low frequency $(100 \mathrm{~Hz}-1 \mathrm{KHz})$ relaxational process was suppressed at $90 \mathrm{MHz}[10]$.

The aim of the presented paper is the complementary dielectric investigation of the relaxation process of GPI crystal at low frequency range up to $1 \mathrm{MHz}$. It seems that more detailed measurements at this range of the frequency allow us to clarify the mechanism of the low frequency dispersion.

\section{Experimental method}

The high quality and transparent crystals GPI were grown by a slow evaporation method from the saturated water solution of stoichiometric quantities of glycine and phosphorus acid at the constant temperature $T=301 \mathrm{~K}$. The main surfaces of the $b$-cut samples were covered with silver electrodes. The mechanically free samples were placed in a nitrogen gas exchange chamber of a cryostat. The temperature in the chamber was controlled with accuracy not worse than $5 \times 10^{-3} \mathrm{~K}$.

The setup allows us to apply an external dc voltage up to $300 \mathrm{~V}$. The measurement of the complex electric permittivity $\varepsilon^{*}(\omega, T)$ was performed in the frequency range of $200 \mathrm{~Hz}-1 \mathrm{MHz}$ using the computer-controlled HP4284A LCR-meter. The field dependence of the electric permittivity at different constant temperatures were obtained under an external bias electric field of an amplitude $300 \mathrm{kV} / \mathrm{m}$ and frequency $2 \times 10^{-3} \mathrm{~Hz}$. 


\section{Results and discussion}

In the most investigated samples of GPI crystals the existence of internal bias electric field has been observed. The existence of this field can be visible during the measurement of the field dependence of electric permittivity at constant temperature above the phase transition or in the field dependence of the temperature at which the maximum of electric permittivity occurs. The maximal value of internal bias field was estimated to be $15 \mathrm{kV} / \mathrm{m}$. From the hysteresis loops the values of the coercive field were calculated. The temperature dependences of the internal bias field and the coercive one are shown in Fig. 1. As it can be seen in Fig. 1, just below the phase transition temperature, both fields increase in the same way and then they are practically temperature independent. It concerns to the narrow temperature range $\left(T_{c}+2.5 \mathrm{~K}\right)$ above the phase transition temperature. The maximum of the bias field is close to the value, which was reported when the nonlinear investigation was performed [3].

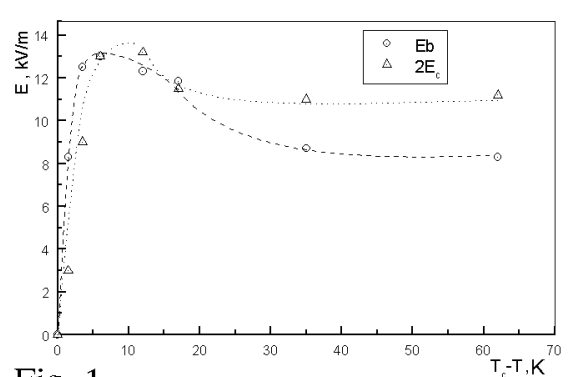

Fig. 1

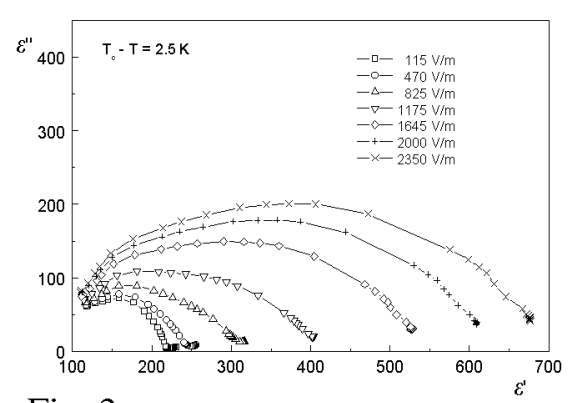

Fig. 2

Fig. 1. The temperature dependence of the bias electric field $\left(E_{\mathrm{b}}\right)$ and the coercive field $\left(E_{\mathrm{c}}\right)$ for GPI crystals.

Fig. 2. The Cole-Cole plots of GPI crystal for different measuring electric fields.

The GPI crystal characterizes the small coercive field (it means the crystal is "electrically soft") so the strong effect of the applied measuring field on the low frequency relaxation process is expected. In Fig. 2. there are presented diagrams of $\varepsilon^{\prime}$ against $\varepsilon^{\prime \prime}$ of the GPI crystal for different measuring electric fields. The results show significant contributions of the domain switching even if a small measuring field is applied.

More detailed information connected with that process was obtained from the measurements of the complex electric permittivity of GPI crystals in the temperature range about $\Delta T=10 \mathrm{~K}$ below $T_{c}$ and in the frequency range of $10^{2}-10^{6} \mathrm{~Hz}$. In Fig. 3. there are presented the dielectric spectra of the low frequency relaxation process for different temperatures in the ferroelectric phase.

The asymmetric shape of the presented spectra suggests the distribution of relaxation processes. The performed computer analysis showed that the obtained 


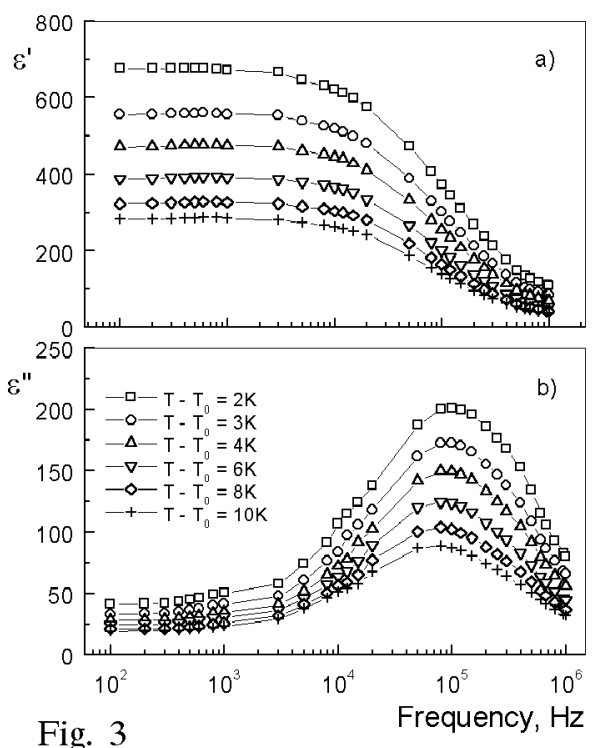

Fig. 3

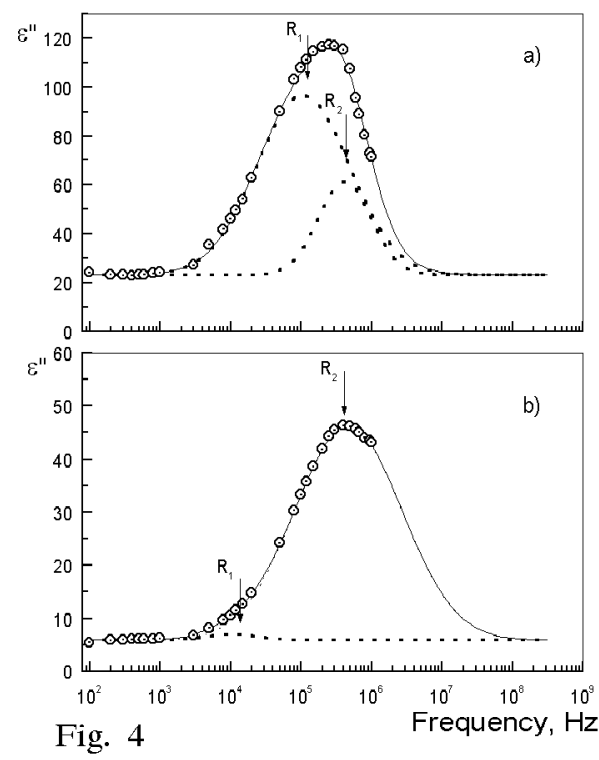

Fig. 4

Fig. 3. The frequency dependence of the real (a) and imaginary (b) part of the electric permittivity of GPI crystal at different temperatures. The value of the measuring ac field was about $2 \mathrm{~V} / \mathrm{cm}$.

Fig. 4. The frequency dependences of the imaginary part of the electric permittivity of GPI crystal for the multidomain (a) and monodomain (b) state.

dielectric spectrum can be fitted by two Debye-type relaxators abbreviated later as $R_{1}$ and $R_{2}$. The first one has a maximum at about $10^{5} \mathrm{~Hz}$ while the second at about $7 \times 10^{5} \mathrm{~Hz}$. The results are presented in Fig. 4, where the mathematical analysis of the dielectric spectrum for multidomain and monodamain sample is shown in Figs. 4a and b, respectively.

In Fig. 4. it is easy to see that under an applied electric field the $R_{1}$ process disappears while the $R_{2}$ one exists in the same place and shape as in a multidomain state. The activation energy of both processes from the temperature dependence of the macroscopic relaxation time was calculated according to Eyring's theory of the reaction process rate. The macroscopic relaxation time of this process is rewritten as follows:

$$
\tau=\tau_{0}\left(\varepsilon_{\mathrm{c}}-\varepsilon_{\infty}\right) \exp (\Delta U / k T)
$$

where $\tau$ and $\tau_{0}$ are macroscopic and microscopic relaxation time, respectively, $\varepsilon_{\mathrm{c}}$ is a maximum value of $\varepsilon^{\prime}, \varepsilon_{\infty}$ is a constant, $\Delta U$ is the activation energy for the reorientation of the electrical dipoles. In our case $\varepsilon_{\infty}$ is equal to 6.4. The value of macroscopic relaxation time (calculated after its saturation near the Curie point) is comparable with that of the hydrogen-bonded ferroelectrics, for example in one-dimensional ferroelectric $\mathrm{CsH}_{2} \mathrm{PO}_{4}$ [12] or TGS-family crystal [13]. In Fig. 5. 


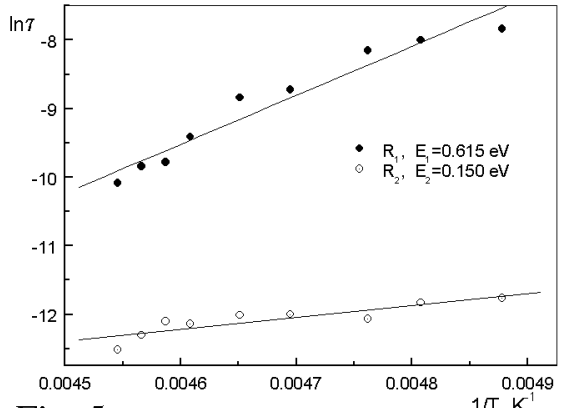

Fig. 5

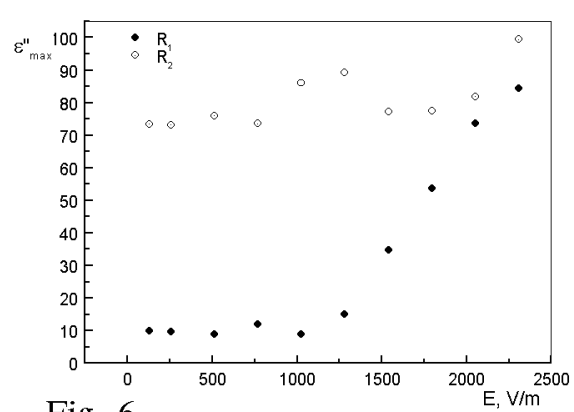

Fig. 6

Fig. 5. The temperature dependence of the relaxation time of the relaxators $R_{1}$ and $R_{2}$.

Fig. 6. Electric field dependence of $\varepsilon_{\max }^{\prime \prime}$ of the relaxators $R_{1}$ and $R_{2}$.

the temperature dependence of the relaxation time $\tau$ is shown. From the slope of $\ln \tau$ vs. $T^{-1}$ the activation energies of the both relaxators $R_{1}$ and $R_{2}$ were found as follows: $E_{1}=0.615 \mathrm{eV}$ and $E_{2}=0.150 \mathrm{eV}$, respectively. The ratio of $E_{1} / E_{2}$ is close to 4.

This kind of behaviour is observed only in the ferroelectric phase and it is obvious to relate it to the presence of the domain structure, which influences the properties of the ferroelectric crystal giving the contribution to its electric permittivity. To decide what is the domain structure contribution to the low-frequency relaxation process, that is to say, which mechanisms can be connected with the presence of two relaxators, the external bias electric field (higher than coercive one) was applied. In Fig. 6 the effect of electric field on the maximal value of $\varepsilon_{\max }^{\prime \prime}$ of the both relaxators is shown. It is seen that when the value of the field is more than coercive one, the relaxator $R_{1}$ appears. This fact indicates that the $R_{1}$ process must be connected with the sideways domain wall movement.

All the models proposed for appearing or reorientation of domain structure are based on the conception of the nucleation character of the process [14-18]. In generally, this process involves all or some of the following steps which may take place: nucleation of isolated nuclei of the reversed state, forward growth of these nuclei through the thickness of the crystal and in the end, sideways expansion of the domain.

The main assumption of nucleation models of the domain wall motion is that its sideways expansion is not a result of a uniform movement of the whole wall parallel to itself but is the result of a more complex nucleation and growth mechanism which occurs next to an already existing wall [15, 18]. The two main quantities have been taken into account while considering the domain structure reorientation: the activation energies (or fields) for the nucleation process of the nuclei and for the sideways expansion of the domain walls. In several works, which have been reported, for instance, to explain the mechanism of polarization reversal, we can find experimentally measured values of these quantities for TGS crystal 
$[14,15,17,19,20]$, Rochelle salt [21] and $\mathrm{BaTiO}_{3}$ [18]. Generally, it must be noticed that the obtained values of the activation fields of the sideways domain wall motion are about four times greater than those for the nucleation process.

The experimental conditions of our experiment is that we have the crystal under two, superimposed electric fields: the bias electric field applied to the crystal to obtain the monodomain state and the small alternating field to measure the dielectric constant. The theoretical consideration of influence of small alternating field on the nucleation of nuclei on domain walls [22] and in random place [23] agrees with the experimental results for TGS and $\mathrm{BaTiO}_{3}$. In particular, it was concluded that the main contribution to the dielectric constant during switching results firstly from the sideways motion of the domain wall and secondly from the alternating field dependence of the growth of residual nuclei in a smaller degree.

From the model of residual nuclei [24] it follows that nuclei are always present in the crystal even in the monodomain sample under an external electric field higher than a coercive one. The reversed domain is formed within the existing residual nuclei because the original domain is unchanged except for this portion of its volume where a reversal nucleus is formed. The appearing of the domain during polarization reversal at the same place of the sample is widely known from direct observations.

\section{Conclusions}

The measurements of the complex electric permittivity of GPI crystals in low frequency range showed that:

- dielectric dispersion in the ferroelectric phase showed two well-distributed relaxational processes $R_{1}$ and $R_{2}$ with activation energies $E_{1}$ and $E_{2}$, respectively,

- the ratio of $E_{1} / E_{2}$ is close to four and it is similar to the ratio of activation fields of domain wall motion and nucleation process,

- the behaviour of found relaxators $R_{1}$ and $R_{2}$ suggest that the first can be connected with the sideways domain wall movement, whereas $R_{2}$ with the influence of measuring, alternating electric field on the growth of the residual nuclei.

\section{References}

[1] M.T. Averbuuch-Pouchot, Acta Crystallogr. C 49, 815 (1993).

[2] S. Dacko, Z. Czapla, J. Baran, M. Drozd, Phys. Lett. A 223, 217 (1996).

[3] R. Tchukvinskyi, R. Cach, Z. Czapla, S. Dacko, Phys. Status Solidi A 165, 309 (1998).

[4] J. Baran, G. Bator, R. Jakubas, M. Śledź, J. Phys., Condens. Matter 8, 10647 (1996) 
[5] B. Kosturek, J. Baran, Ferroelectrics Lett. 27, 11 (2000).

[6] G.A. Samara, in: Advances in High Pressure Research, Ed. R.S. Bradley, Vol. 3, Academic Press, New York 1969, p. 155.

[7] N. Yasuda, A. Keneda, Z. Czapla, J. Phys, Condens. Matter 9, L447 (1997).

[8] J. Furtak, Z. Czapla, A.V. Kityk, Z. Naturforsch. A 52, 778 (1997).

[9] J. Tritt-Goc, N. Piślewski, L. Szczepańska, R. Goc, Solid State Commun. 108, 189 (1998).

[10] R. Tchukvinskyi, Z. Czapla, R. Sobiestianskas, A. Brilingas, J. Grigas, J. Baran, Acta Phys. Pol. A 92, 1191 (1997).

[11] R. Sobiestianskas, A. Brilingas, Z. Czapla, J. Korean Phys. Soc. 32, S377 (1998).

[12] E. Kanda, A. Tamaki, T. Fuimura, J. Phys. C, Solid State Phys. 15, 3401 (1982).

[13] K. Takayama, K. Deguchi, E. Nakamura, J. Phys. Soc. Jpn. 53, 4121 (1984).

[14] C.F. Pulvari, W. Kuebler, J. Appl. Phys. 29, 1742 (1958).

[15] R.C. Miller, G. Weinreich, Phys. Rev. 117, 1460 (1960).

[16] E. Fatuzzo, Phys. Rev. 127, 1999 (1962).

[17] H.H. Wieder, J. Appl. Phys. 35, 1224 (1964).

[18] A. Fouskova, Czech. J. Phys. B 20, 790 (1970).

[19] N. Nakatani, J. Phys. Soc. Jpn. 32, 1556 (1972).

[20] B. Hilczer, Thesis, Polish Acad. Sci., Institute of Physics, Warsaw 50, 1975.

[21] G. Nogami, J. Phys. Soc. Jpn. 37, 153 (1974).

[22] E. Fatuzzo, W.J. Merz, Ferroelectricity, North-Holland P.C., Amsterdam 1967.

[23] A. Fouskova, J. Phys. Soc. Jpn. 20, 1625 (1965).

[24] J. Janta, J. Phys. Soc. Jpn. Suppl. 28, 340 (1970). 